Revista Electrónica Complutense de Investigación en Educación Musical ISSNe: 1698-7454

https://dx.doi.org/10.5209/reciem.62409

\title{
Aprendizaje-servicio en educación musical: revisión de la literatura y recomendaciones para la práctica ${ }^{1}$
}

\author{
Oscar Chiva-Bartoll ${ }^{2}$, Celina Salvador-García ${ }^{3}$, Sergio Ferrando-Félix ${ }^{4}$, Alberto Cabedo-Mas ${ }^{5}$
}

Recibido: 10/12/2018 / Aceptado: 31/03/2019

Resumen. Con la intención de promover una educación musical que responda a los desafíos actuales, están empezando a emerger con fuerza posicionamientos educativos sociocomunitarios que incluyen el Aprendizaje-Servicio (ApS) como propuesta metodológica. A fin de explorar las posibilidades y limitaciones del ApS en educación musical, la presente revisión de la literatura parte del objetivo de proveer a la comunidad académica de las herramientas y conocimientos necesarios para establecer el estado del arte y optimizar este tipo de praxis educativas. Teniendo como referencia las pautas establecidas por Moher, Liberati, Tetzlaff, y Altman (2009), la revisión parte de una búsqueda en tres de las bases de datos con mayor reconocimiento científico a nivel internacional en el área de educación: Web of Science (WoS), Scopus-Elvesier (SCOPUS) y Education Resources Information Center (ERIC).Los criterios de inclusión y exclusión utilizados permitieron eliminar aquella información vinculada con la búsqueda pero ajena a los objetivos e intereses del presente estudio, dotando al trabajo de mayor rigurosidad. El análisis de los 18 estudios que superaron el proceso de selección comprende los siguientes ejes descriptivos: método de investigación, instrumentos de investigación utilizados, contenido curricular trabajado, etapa educativa, receptores del servicio, impacto generado sobre los receptores, impacto en el alumnado, duración de los proyectos y modalidad de aprendizaje-servicio. En el texto se analizan uno a uno estos ejes concluyendo, a partir de los resultados obtenidos, con recomendaciones concretas de aplicación e investigación que invitan a abordar, de un modo fundamentado, la educación musical sociocomunitaria a través del ApS.

Palabras clave: aprendizaje sociocomunitario; aprendizaje-servicio; revisión sistemática; música; método pedagógico.

\section{[en] Service Learning in Musical Education: Literature Review and Recommendations for Practice}
Abstract. In order to meet the needs of nowadays challenges, music education with a social impact is starting to gain attention, for example through sociocommunitary educational projects that include Service Learning (SL) as a methodological proposal. To explore the possibilities and limitations of SL in musical education, the present review attempts to provide the academic community with those tools and knowledge which are necessary in order to establish the state of the art and optimize this type of educational practices. Based on the guidelines established by Moher et al. (2009), the starting point of this review is a search developed in three of the most important databases of the educational field from an

\footnotetext{
1 Proyecto EDU2017-86311-P (MINECO): Análisis multidimensional del impacto socioeducativo de iniciativas musicales comunitarias y de aprendizaje-servicio en la escuela: el programa MUSIQUEM.

$2 \quad$ Universitat Jaume I, ochiva@uji.es

3 Universitat Jaume I, salvadoc@uji.es

$4 \quad$ Universitat de València, serfefe@uv.es

$5 \quad$ Universitat Jaume I, cabedoa@uji.es
} 
international perspective: Web of Science (WoS), Scopus-Elvesier (SCOPUS) and Education Resources Information Center (ERIC). The inclusion and exclusion criteria utilized let the research team remove the information that, despite being linked to the topic, was not within the objectives and interests of the study. The analysis comprised the 18 papers that satisfied the criteria, and comprises the following descriptive axes: research method, research tools used, curricular content developed, educational stage, service receivers, impact on the receivers, impact on the students, length of the projects and type of service-learning. The text analyzes each one of these axes. Regarding the results obtained, this paper concludes with several concrete recommendations to assist both practical application and scientific investigation. These suggestions aspire to foster the sociocummunitary educational possibilities of musical education through SL.

Keywords: socio-community learning; service-learning; systematic review; music; pedagogical method.

Cómo citar: Chiva-Bartoll, O.; Salvador-García, C.; Ferrando-Félix, S. y Cabedo-Mas, A. (2019). Aprendizaje-servicio en educación musical: revisión de la literatura y recomendaciones para la práctica. Revista Electrónica Complutense de Investigación en Educación Musical, 16, 57-74.

\section{Introducción}

Diversas cuestiones mantienen viva la llama de un debate que lleva a la educación musical a un conflicto evidente polarizado entre el deseo de que la música represente un lugar central en el currículo vigente y el papel secundario al que la ha relegado muchas veces una práctica docente muy técnica y desligada de los intereses del alumnado (Siebenaler, 2005). Nuevos posicionamientos están empezando a emerger con fuerza, intentando promover una idea de educación musical que responda a los retos actuales. Algunas de estas propuestas incluyen iniciativas de educación musical sociocomunitaria. Entender la educación musical como un proyecto socioeducativo implica un modelo educativo comprometido con el aprendizaje de la música como fin en sí mismo (Cabedo-Mas, 2014), pero también como medio para el desarrollo de competencias centradas en aprender a vivir y convivir (Vicente y Azorín, 2013). Para ello es requisito establecer propuestas que abran las puertas del aula de música e incluyan iniciativas musicales comunitarias (Casacuberta, Rubio y Serra, 2011; Higgins, 2012). Estas iniciativas, tal como describe la International Society for Music Education (2013), van más allá de involucrar a los participantes en la creación musical; les proporcionan oportunidades para construir expresiones personales y comunitarias de inquietudes artísticas, sociales, políticas y culturales. Estas actividades, vehiculadas en no pocas ocasiones a través de métodos pedagógicos activos y experienciales, pueden contribuir a la mejora de la calidad de vida de las comunidades, fomentando y capacitando a los participantes para que se conviertan en agentes encargados de extender y desarrollar la música en sus comunidades. Al mismo tiempo, la música, como lenguaje emocional, posee unas características específicas que, correctamente gestionados, tienen grandes potencialidades para generar espacios de conexión intersubjetiva entre las personas. La participación en experiencias musicales positivas es una herramienta para promover aprendizajes conceptuales significativos, pero también para el desarrollo de competencias centradas en aprender a vivir y a convivir (CabedoMas y Díaz-Gómez, 2013). Para ello, es necesario impulsar métodos educativos en educación musical que no sólo promuevan el aprendizaje en la música, sino también a través de la música.

Uno de los métodos de aprendizaje activo y experiencial que más se está extendiendo en los últimos tiempos es el Aprendizaje-Servicio (ApS), proporcionando al alumnado las condiciones necesarias para aprender autónomamente a la vez que resuelve necesi- 
dades o injusticias sociales. El ApS tiene un innegable carácter experiencial, centrado en actuar en contextos reales que, a todas luces, resultan más motivantes que los espacios educativos tradicionales (Miedl y Sulentic, 2018; Gil-Gómez, Moliner-García, ChivaBartoll y García, 2016). Su aplicación combina procesos de aprendizaje y de servicio, basados en la adquisición y comprensión reflexiva de contenidos curriculares en contextos reales, de forma activa y experiencial, con el objetivo de mejorar la capacidad crítica a la vez que se ofrece un beneficio social (Chiva-Bartoll, Pallarès-Piquer, Gil-Gómez, 2018; Furco y Billig, 2002).

El ApS cuenta con una serie de elementos particulares que conforman su esencia: a) parte de una vinculación curricular necesaria con la materia en la que se aplica; b) debe existir reciprocidad, entendida como la necesidad de integrar, equilibrar y evaluar los objetivos de aprendizaje y los de servicio (Anderson, Swick e Yff, 2001); c) debe existir reflexión, centrada en interiorizar los aprendizajes que emanan de la experiencia y en incentivar al alumnado a conocerse a sí mismo; d) la toma de decisiones del alumnado debe estar presente en todas las fases del proceso (diseño, ejecución y evaluación); e) debe prestarse atención a una necesidad o injusticia real, es decir, los programas de ApS no pueden quedarse en planificaciones hipotéticas o meras declaraciones de intenciones.

En cuanto a sus posibilidades de aplicación, una gran particularidad del ApS es la riqueza y diversidad que este permite. A partir de las clasificaciones más extendidas ofrece cuatro modalidades de actuación: servicio directo, servicio indirecto, denuncia y servicio de investigación (Furco, 1996; 2002). El servicio directo plantea una interacción directa entre el alumnado y el colectivo receptor. Algunos ejemplos en este ámbito podrían materializarse en la impartición de talleres o clases sobre contenidos propios del área a colectivos necesitados. El servicio indirecto, por su parte, responde a aquellos proyectos que prescinden del contacto personal con los beneficiarios. Es decir, a través del servicio indirecto se aportan principalmente recursos, ideas, planes de actuación, etc., para que el colectivo desfavorecido pueda hacer uso de ellos. En este caso, el alumnado de educación musical podría generar materiales, composiciones, etc., que finalmente emplearían los colectivos desfavorecidos. En tercer lugar, el modelo de denuncia invita a realizar campañas de concienciación y sensibilización social. Algunos ejemplos en el ámbito de la educación musical podrían centrarse en crear campañas de denuncia respecto a temas como el sexismo, la xenofobia, etc., aprovechando vías de difusión al alcance del alumnado. Finalmente, el servicio de investigación, consiste en recopilar información en torno a necesidades, con el objeto de sistematizarla y hacerla más accesible a la comunidad. Por ejemplo, tratar de analizar de manera crítica determinadas problemáticas propias del mundo de la música y la cultura para hacerlas más accesibles a colectivos sociales próximos. (Salvador-García y Ferrando-Félix, 2018).

La apuesta por el ApS se está viendo incrementada en multitud de áreas y niveles educativos (Warren, 2012; Yorio y Ye, 2012), siendo abundantes los estudios que analizan su impacto (Novak, Markey y Allen, 2007). Este rápido crecimiento ha sido posible gracias al trabajo y determinación de docentes e investigadores comprometidos con un movimiento comunitario que, en general, se nutre y está próximo a prácticas de educación activa y experiencial (Butin, 2006 Chiva-Bartoll, Capella-Peris y Pallarès-Piquer, 2018), entre los que no faltan representantes en la educación musical (Feen-Calligan y Matthews, 2016). La mayor parte de aplicaciones e investigaciones de ApS en educación derivan de la etapa universitaria, circunscribiéndose muchas de estas experiencias al campo de la formación inicial docente. Al respecto, existen evidencias sobre los efectos positivos que proporciona el ApS, especialmente en lo que concierne al alumnado que 
lo aplica (Conway, Amel y Gerwien, 2009). En este sentido, Butin (2003) plantea un modelo explicativo ampliamente aceptado de sus efectos según el cual el ApS incide en el estudiantado desde cuatro perspectivas: la técnica, la cultural, la política y la postestructural. Por un lado, la perspectiva técnica se focaliza en la efectividad pedagógica, a menudo establecida en base a elementos innovadores como la calidad o la eficiencia de los aprendizajes (Seban, 2013). La segunda categoría es la cultural, y se centra en el desarrollo de habilidades y valores para con la comunidad social, así como con una mayor comprensión de la diversidad; generando, a menudo, un incremento de la responsabilidad social de los participantes. En la perspectiva política, el ApS parece ofrecer al alumnado una nueva visión del mundo, haciéndole consciente de cómo ciertos grupos sociales dominan sobre otros y, por tanto, cómo se establecen las relaciones de poder en la sociedad. Por último, la perspectiva post-estructural, se centra en cómo el ApS produce cambios en los propios participantes gracias a su capacidad de generar nuevas concepciones sobre el mundo y sobre uno mismo.

En el ámbito educativo en general trabajos relativos al ApS como los meta-análisis de Conway et al. (2009), Warren (2012) y Yorio y Ye (2012) ofrecen una aproximación panorámica de su potente crecimiento en términos de investigación e implementación. No obstante, en el punto de proliferación en el que nos encontramos, tanto en la educación musical como en el resto de campos disciplinares empiezan a ser necesarias revisiones más específicas (Novak et al., 2007; Warren, 2012). En el ámbito que nos ocupa, no cabe duda de que la música representa un elemento artístico-cultural a través del cual los colectivos implicados en los programas de ApS comparten espacios de interacción, conexión y crecimiento personal (Cabedo-Mas y Díaz-Gómez, 2013). Este hecho, característico de la educación musical, podría reforzar los efectos positivos generados por el ApS. En consecuencia, la presente revisión parte del objetivo de proveer a la comunidad científica y académica de las herramientas y conocimientos necesarios para conocer y generar nuevas experiencias e investigaciones de ApS en educación musical.

\section{Método}

\subsection{Estrategia de búsqueda}

Teniendo como referencia las pautas establecidas por Moher et al (2009), la revisión parte de una búsqueda en tres de las bases de datos con mayor reconocimiento científico a nivel internacional en el área de educación: Web of Science (WoS), Scopus-Elvesier (SCOPUS) y Education Resources Information Center (ERIC).

La búsqueda se desarrolló entre los meses de enero y marzo de 2018. Se tomaron aquellas publicaciones que relacionaban el $\mathrm{ApS}$ con la educación musical. Para ello, se realizó una búsqueda sistemática que incluyera en el título, palabras clave o resumen, los siguientes términos conectados mediante el operador booleano "and": para el primer campo se utilizó la terminología service-learning y service learning; para el segundo campo, se utilizaron los términos music y musical practice. Estos términos fueron incorporados en todas las combinaciones posibles.

\subsubsection{Criterios de inclusión y exclusión}


Los criterios de inclusión y exclusión utilizados permitieron eliminar aquella información vinculada con la terminología empleada pero ajena a los objetivos del presente estudio, dotando a la revisión de una mayor rigurosidad. En primer lugar, se acotaron los idiomas de las publicaciones, optando por el inglés y el español a fin de evitar textos ininteligibles para el equipo investigador. En segundo lugar, se determinó un intervalo de años comprendido entre 2003 y 2017, ambos incluidos. Situamos el inicio en esta fecha para obtener información de aquello que se ha estudiado en los últimos 15 años, ya que el ApS ha ido evolucionando y expandiéndose especialmente a lo largo de este periodo. En tercer lugar, siguiendo criterios de eficiencia y relevancia, se ha utilizado el filtro del "tipo de documento" o documenttype, limitando la búsqueda a artículos, libros o capítulos de libro. En cuarto lugar, se han filtrado los resultados en función del "área de conocimientos" o subjectarea para evitar aquellos artículos que hablaban de conceptos relacionados con la búsqueda, pero desde perspectivas no educativas. Por ello, en WoS se concretó la búsqueda a las áreas de Psychology, BehaviouralSciences, Music, Education, EducationalResearchyArts and Humanities. Por su parte, en SCOPUS se han escogido los campos de conocimiento que engloban Psychology, Social Sciences y Arts and Humanities. En el caso de ERIC, la búsqueda ha sido llevada a cabo de forma generalizada ya que no permite restringir los resultados por campos de conocimiento. Finalmente, fueron excluidos aquellos textos que abordaban la música desde un enfoque terapéutico o relacionado con la musicoterapia, a fin de seleccionar estrictamente textos de índole educativa.

\subsubsection{Fases o procedimiento de revisión}

Dentro del procedimiento de búsqueda la primera fase, o identificación, fue llevada a cabo por tres investigadores, que de forma autónoma realizaron búsquedas independientes coincidiendo sus hallazgos en todos los casos. Seguidamente, el equipo investigador aplicó los criterios de exclusión establecidos a los 55 artículos obtenidos. Una vez aplicados los criterios, únicamente 18 artículos cumplían con los requisitos. Seguidamente, se procedió a la implementación de la estrategia bola de nieve, consistente en analizar estudios adicionales identificados en las referencias de las fuentes de la búsqueda inicial. Mediante este procedimiento se analizaron un total de 11 nuevas publicaciones. Sin embargo, ninguna de ellas acabó cumpliendo con los criterios de inclusión, por lo que no variaron el resultado y siguieron siendo 18 los estudios que acabaron superando la fase de cribado. A continuación, se procedió a la fase de idoneidad, consistente en analizar los textos completos para valorar su adecuación a los objetivos de la revisión. Como todos ellos se ajustaban a la misma, en la fase final de inclusión se tuvieron en cuenta el total de 18 artículos. La Figura 1 muestra gráficamente este flujo de búsqueda.

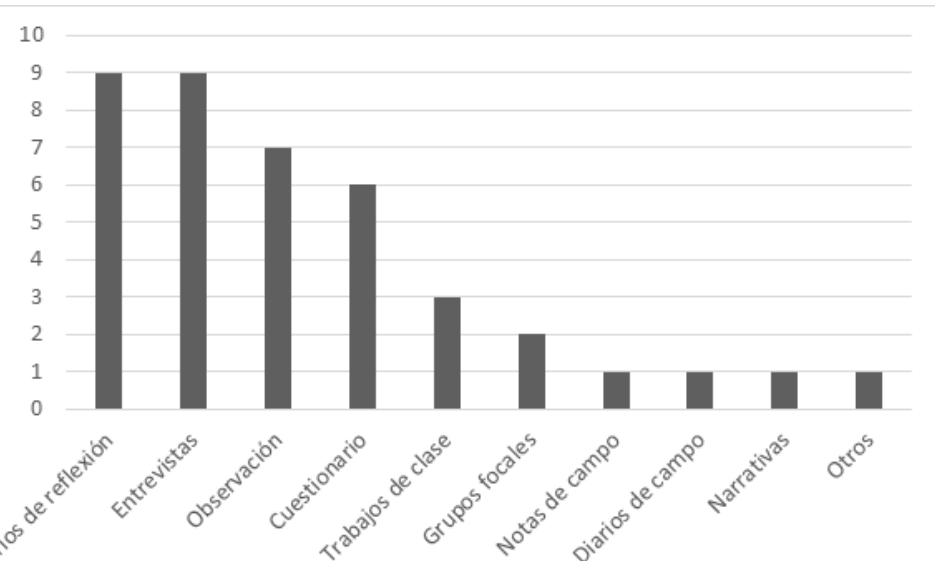


Figura 1. Instrumentos empleados para analizar efectos del ApS en educación musical.

\section{Resultados}

El análisis de los estudios seleccionados se articula en torno a los siguientes ejes descriptivos: método de investigación, instrumentos de investigación utilizados, contenido curricular trabajado, etapa educativa, receptores del servicio, impacto social, impacto en el alumnado (analizado desde las perspectivas de Butin (2003) anteriormente mencionadas, a saber, técnica, cultural, política y post-estructural), duración de los proyectos y tipo de servicio (directo, indirecto, denuncia e investigación). Los resultados principales, que pasamos a describir a continuación, quedan resumidos en la Tabla 1. 


\begin{tabular}{|c|c|c|c|c|c|c|c|c|c|c|c|c|}
\hline$\stackrel{\varrho}{:}$ & $\begin{array}{l}\stackrel{0}{0} \\
\text { : }\end{array}$ & 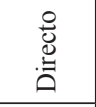 & 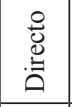 & $\begin{array}{l}\stackrel{0}{0} \\
\stackrel{0}{0}\end{array}$ & 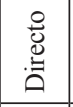 & 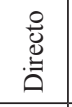 & , & , & $\begin{array}{l}\stackrel{0}{0} \\
.00 \\
.00\end{array}$ & 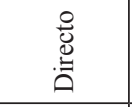 & 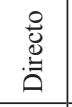 & 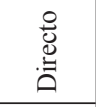 \\
\hline & 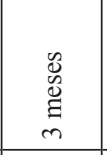 & 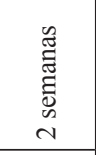 & 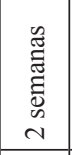 & 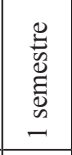 & 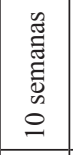 & 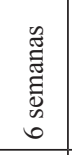 & 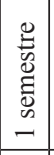 & 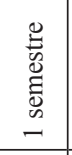 & 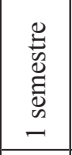 & 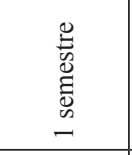 & 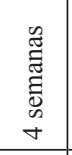 & 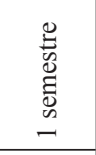 \\
\hline 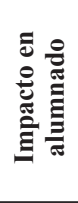 & 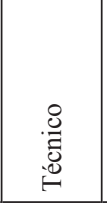 & 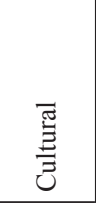 & 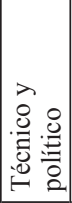 & 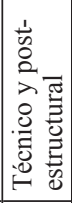 & 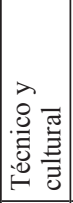 & . & 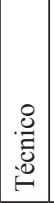 & . & 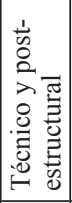 & 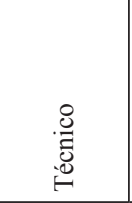 & 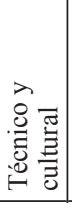 & 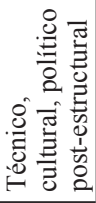 \\
\hline 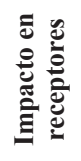 & , & , & 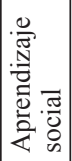 & 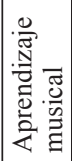 & , & , & , & , & I & 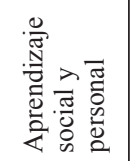 & , & , \\
\hline 密 & 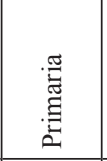 & 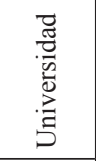 & 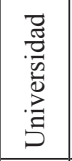 & 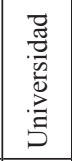 & 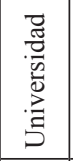 & 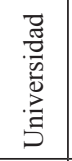 & 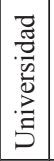 & 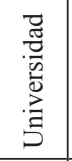 & 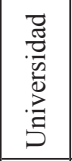 & 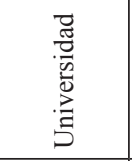 & 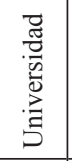 & 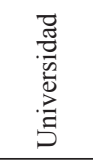 \\
\hline 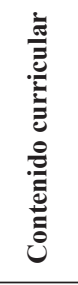 & , & 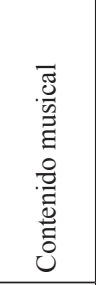 & 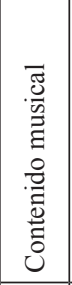 & 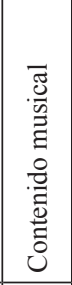 & 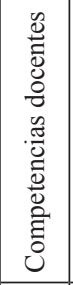 & 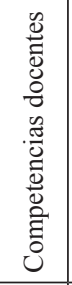 & 1 & , & , & 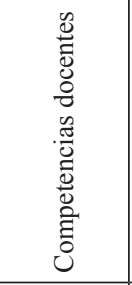 & 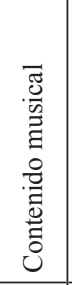 & 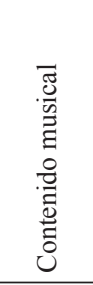 \\
\hline 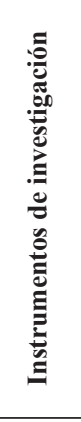 & 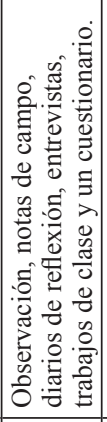 & 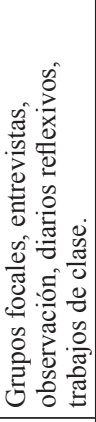 & 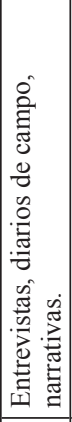 & 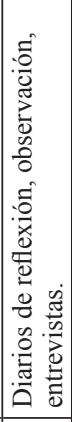 & 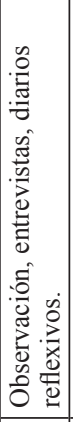 & , & 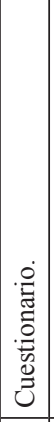 & 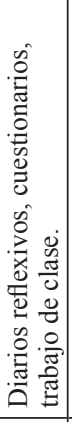 & & 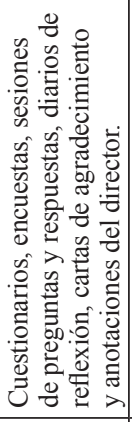 & 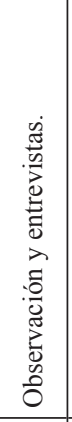 & 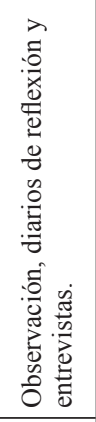 \\
\hline 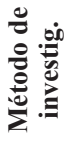 & 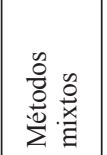 & 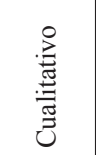 & : & 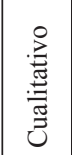 & 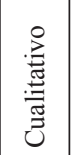 & 苞 & 胥 & . & 胥 & 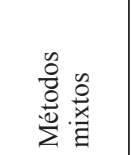 & 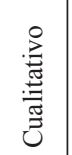 & 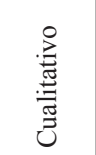 \\
\hline 黄 & 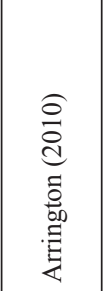 & 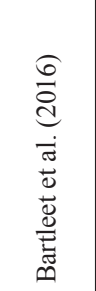 & 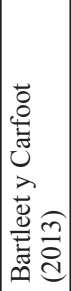 & 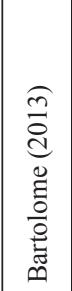 & 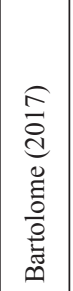 & 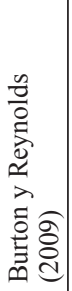 & 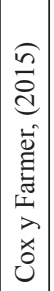 & 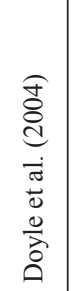 & 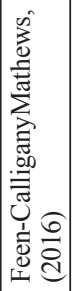 & 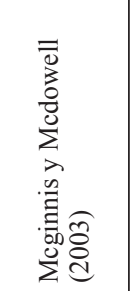 & 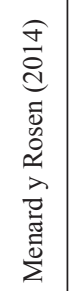 & 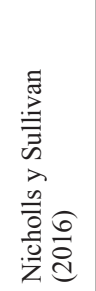 \\
\hline
\end{tabular}




\begin{tabular}{|c|c|c|c|c|c|c|}
\hline$\stackrel{\varrho}{\nexists ٍ]}$ & $\begin{array}{l}\stackrel{8}{\mathscr{U}} \\
\stackrel{\mathscr{U}}{\Delta}\end{array}$ & 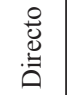 & $\begin{array}{l}\stackrel{0}{0} \\
\stackrel{0}{0.0}\end{array}$ & 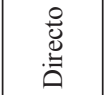 & $\begin{array}{l}\stackrel{0}{0} \\
\stackrel{0}{0.0}\end{array}$ & , \\
\hline 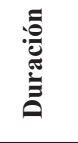 & 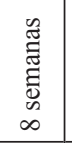 & 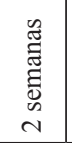 & 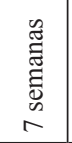 & $\begin{array}{l}\text { 怘 } \\
\text { 㫕 } \\
+\end{array}$ & 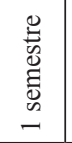 & 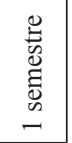 \\
\hline 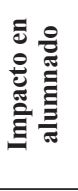 & 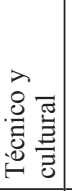 & 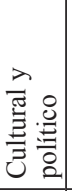 & 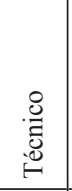 & 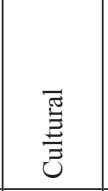 & 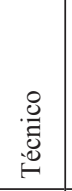 & 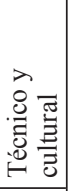 \\
\hline 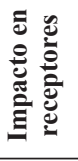 & 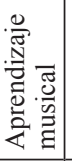 & 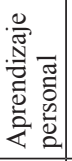 & 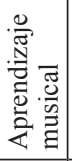 & 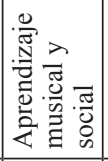 & 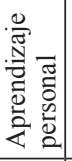 & 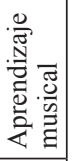 \\
\hline 穿䒿 & 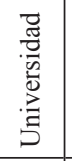 & 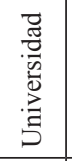 & 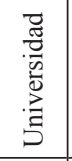 & 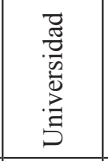 & 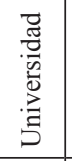 & 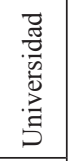 \\
\hline 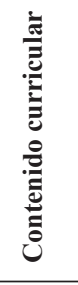 & 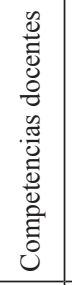 & 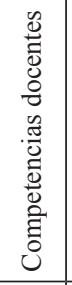 & 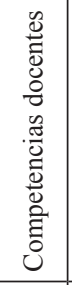 & 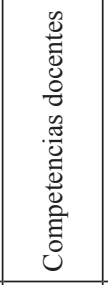 & 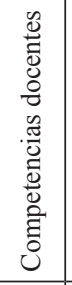 & 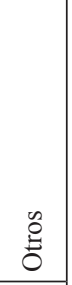 \\
\hline 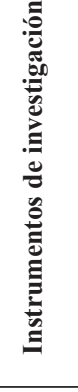 & 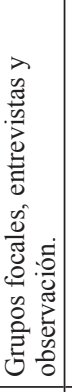 & 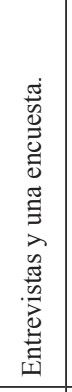 & 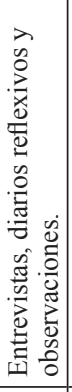 & 1 & 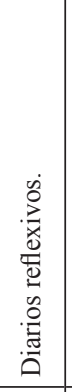 & 1 \\
\hline 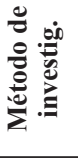 & 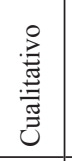 & : & 䔍 & ' & 䔍 & 1 \\
\hline & $\begin{array}{l}\text { E } \\
\stackrel{2}{d} \\
0 \\
\frac{a}{2}\end{array}$ & 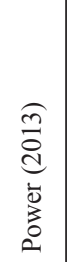 & 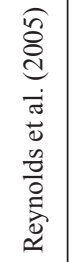 & 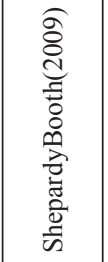 & 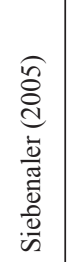 & 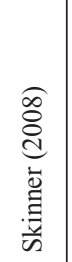 \\
\hline
\end{tabular}




\subsection{Métodos de investigación}

Los resultados nos dan a conocer que las investigaciones centradas en la educación musical y el ApS han sido llevadas a cabo principalmente siguiendo métodos cualitativos (Bartleet, Sunderland y Carfoot, 2016; Bartleet y Carfoot, 2013; Bartolome, 2013; Bartolome, 2017; Burton y Reynolds, 2009). Se trata en muchos casos de trabajos en los que se valora el desarrollo de valores personales y sociales del alumnado participante. Por otra parte, aunque con variables similares, son pocos los estudios que se abordan desde los métodos mixtos (Arrington, 2010; McGinnis y McDowell, 2003) y solamente uno que se afronta exclusivamente desde un enfoque cuantitativo (Cox y Farmer, 2014).

\subsection{Instrumentos de investigación}

Como consecuencia directa del método de investigación cualitativa mayoritariamente empleado, observamos en la figura 2 cómo los instrumentos de investigación más empelados son los diarios de reflexión, las entrevistas y la observación. En menor medida se utilizan otros instrumentos de índole cualitativa como los grupos focales o los propios trabajos de clase, siendo los menos empleados los instrumentos de carácter cuantitativo.

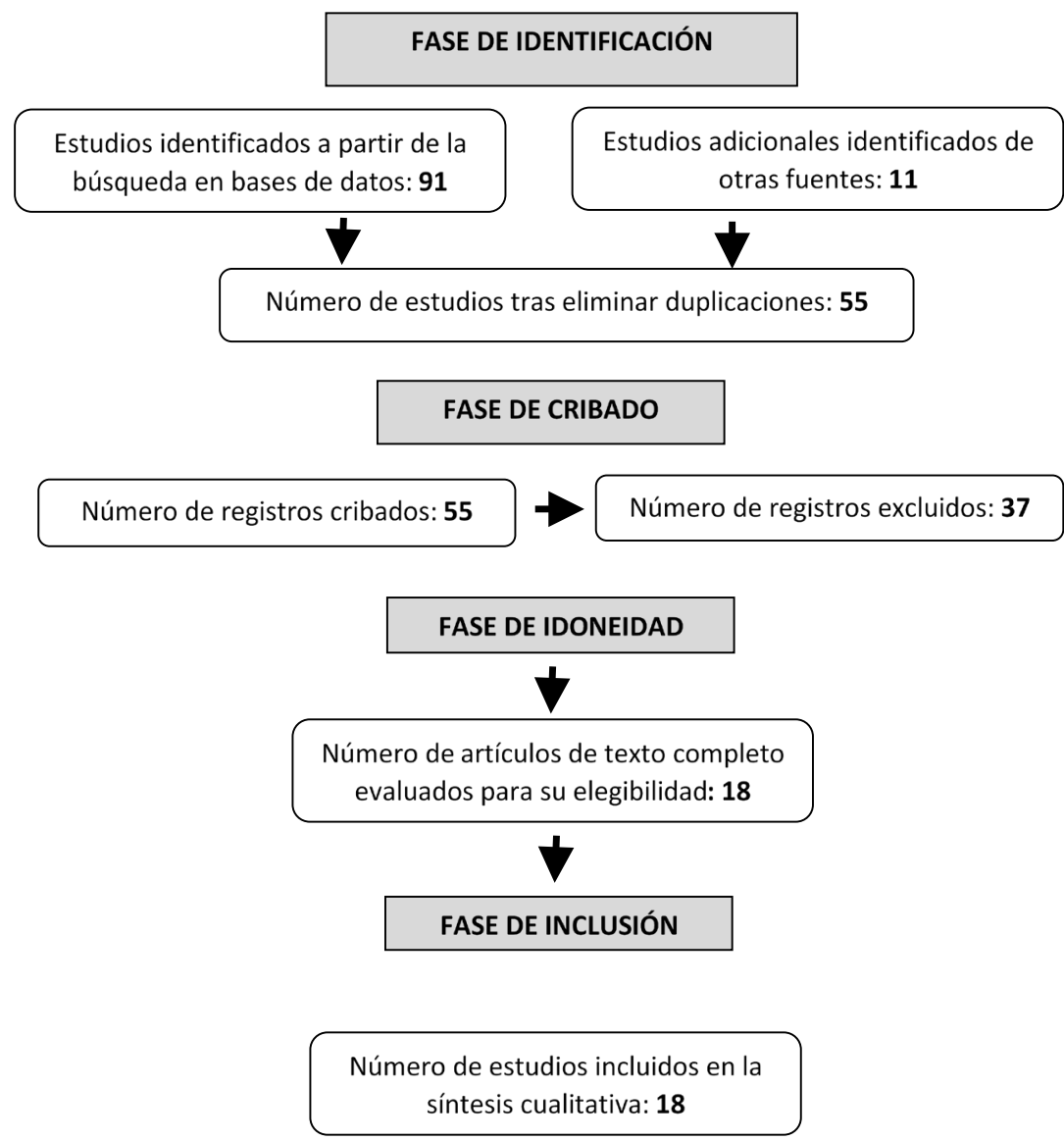

Figura 2. Flujo de búsqueda. (Adaptado de Moher et al., 2009). 


\subsection{Contenidos curriculares trabajados}

Los contenidos que han sido trabajados en mayor medida en los proyectos de ApS se ajustan a los dos ejes temáticos de la investigación. Es decir, por un lado encontramos diversos estudios que analizan de manera directa los aprendizajes musicales (Bartleet et al., 2016; Bartleet y Carfoot, 2013; Bartolome, 2013; Menard y Rosen, 2014; Nicholls y Sullivan, 2016). Por otro lado, al trabajar a partir de la metodología ApS, algunas de las investigaciones han centrado esfuerzos en analizar la adquisición de competencias personales y sociales o de competencias docentes (en el caso de aplicaciones en formación del profesorado) que pueden ser trabajadas y adquiridas a lo largo del proceso (Bartolome, 2017; Burton y Reynolds, 2009; McGinnis y McDowell, 2003; Pike, 2017; Power, 2013; Reynolds, Jerome, Preston, y Haynes, 2005; Shepard y yBooth, 2009; Siebenaler, 2005).

\subsection{Etapas educativas estudiadas}

En cuanto a las etapas a las que van dirigidos los estudios, existe un predominio casi total del periodo universitario. De hecho, excepto una de las publicaciones que alude a la fase de Educación Primaria (Arrington, 2010), el resto de proyectos examinados han sido implementados por alumnado de Educación Superior (especialmente desde el Área de Didáctica de la Música). Además, la totalidad de estos estudios han sido encontrados en lengua inglesa y procedentes en su mayoría de los Estados Unidos, a excepción de tres de ellos que han sido llevados a cabo en Australia.

\subsection{Colectivos receptores del servicio}

Los colectivos receptores del servicio han sido muy variados. Podemos diferenciar entre los grupos formados previamente a la implementación del proyecto y los creados y diseñados para tal finalidad. Por una parte, dentro de los formados previamente, encontramos receptores como personas mayores de un asilo (Arrington, 2010), grupos de educación infantil y primaria (Bartolome, 2013; Bartolome, 2017; Burton y Reynolds, 2009; Power, 2013; Reynolds et al., 2005), centros comunitarios (Feen-Calligan y Mathews, 2016), escuelas rurales (McGinnis y McDowell, 2003), jóvenes enrolados en programas de composición musical (Menard y Rosen, 2014), centro de detección juvenil (Nicholls y Sullivan, 2016) y organizaciones (Siebenaler, 2005). Por otra parte, dentro de los grupos creados podemos encontrar artistas aborígenes indígenas y no indígenas (Bartleet et al., 2016), músicos indígenas (Bartleet y Carfoot, 2013), adolescentes que no podían pagar una escuela de música (Pike, 2017) y niños y niñas sin hogar (Shepard y Booth, 2009).

\subsection{Impacto generado sobre los receptores}

Con respecto al impacto que el ApS ha suscitado sobre los colectivos receptores del servicio social el análisis refleja, por una parte, el desarrollo de una vertiente social, relacionada con la promoción de valores cívicos y de convivencia (Bartleet y Carfoot, 2013); y, por otra parte, una vertiente individual, relativa a dimensiones como la transformación y el crecimiento personal (Power, 2013; Siebenaler, 2005). Además, estas dos vertientes han llegado a ser potenciadas en algunos casos de manera conjunta (Mcginnis y Mcdowell, 2003). De un modo más específico e intrínsecamente ligado a los anteriores, también hay investigaciones que conectan el impacto comunitario del ApS 
con el desarrollo de conocimientos ligados específicamente al campo musical (Bartolome, 2013; Pike, 2017; Reynolds et al., 2005; Shepard y Booth, 2009; Skinner, 2008).

\subsection{Impacto generado en el alumnado que aplica los programas}

El impacto generado sobre el alumnado que lleva a cabo la experiencia de ApS ha sido analizado en función de las dimensiones previamente descritas, a saber, técnica, cultural, política y post-estructural (Butin, 2003). Todas ellas se ven reflejadas en varios artículos de la muestra e, incluso en diversas ocasiones, encontramos ejemplos que combinan varias de estas opciones. Por ejemplo, técnica y política (Bartleet y Carfoot, 2013) o técnica y post-estructural (Bartolome, 2013). En general, de las cuatro perspectivas citadas, aquella que ha tenido una mayor presencia o relevancia ha sido la vertiente técnica (Arrington, 2010; Bartleet y Carfoot, 2013; Bartolome, 2013; Bartolome, 2017; Cox y Farmer, 2014; McGinnis y McDowell, 2003). Además, resulta interesante mencionar que las perspectivas política y post-estructural han aparecido siempre compartiendo análisis con alguna otra categoría, nunca de forma exclusiva.

\subsection{Duración de los proyectos}

En cuanto a la duración de los programas, vemos que la mayor parte duran un semestre académico (Cox y Farmer, 2014; Doyle, Hotchkiss, Noel, Huss y Holmes, 2004; Feen-Calligan y Mathews, 2016; Mcginnis y Mcdowell, 2003; Siebenaler, 2005; Skinner, 2008). En menor medida, pero representando una muestra no despreciable, también encontramos aplicaciones que oscilan entre una y ocho semanas (Bartleet et al., 2016; Bartleet y Carfoot, 2013; Power, 2013). Finalmente, encontramos un único estudio longitudinal de cuatro años de duración (Shepard y Booth, 2009).

\subsection{Modalidad de aprendizaje-servicio}

Respecto a la modalidad de ApS aplicada, vemos que la totalidad de los estudios aluden al servicio directo, no habiéndose encontrado referencias al servicio indirecto, a la denuncia ni tampoco al servicio investigación. En este sentido, mediante la modalidad de servicio directo, son múltiples las actividades que han sido llevadas a cabo. La acción más recurrente ha sido el desarrollo de prácticas docentes relativas a la educación musical (Bartolome, 2013; 2017; Nicholls y Sullivan, 2016; Pike, 2017; Power, 2013; Reynolds et al., 2005). También se repite en diversos textos la realización de actividades creativas o talleres vinculados a algún elemento musical concreto (McGinnis y McDowell, 2003; Menard y Rosen, 2014; Shepard y Booth, 2009; Siebenaler, 2005). Por su parte, Arrington (2010) y Burton y Reynolds (2009) exponen el desarrollo de representaciones musicales de diversas índoles, mientras que Bartleet el al., (2016) y Bartleet y Carfoot (2013) comentan la realización de actividades variables que pueden incluir desde la instalación de un estudio de grabación hasta la gestión de un festival comunitario.

\section{Discusión}

En el presente apartado se presenta una interpretación de los resultados atendiendo a las principales conexiones entre las variables analizadas. Esta reflexión aportará 
claves para comprender mejor cómo los factores culturales y contextuales de cada sociedad podrían influir en la aplicación del ApS.

Reparando en el contexto de desarrollo de la investigación, la muestra analizada presenta una dicotomía clara, por un lado, aparecen tres artículos realizados en Australia (Bartleet y Carfoot, 2013; Bartleet et al., 2016; Power, 2013) mientras que el resto provienen de los Estados Unidos de América. A tenor de los resultados obtenidos en estas publicaciones, parece que el entorno puede repercutir en algunas características de los programas de ApS desde la educación musical. Por ejemplo, se detecta que los tres programas australianos son los más cortos. En concreto estos se llevaron a cabo de forma intensiva durante un período de dos semanas. Dicha duración se ve relacionada, asimismo, con el factor de que dichas iniciativas se han desarrollado en lugares alejados de donde habita el estudiantado implicado. Es decir, el alumnado participante en los estudios de Bartleet y Carfoot (2013) y Bartleet et al. (2016) debía desplazarse desde Queensland hasta Central Australia para ejecutar sus respectivos proyectos de ApS, mientras que el servicio prestado en el estudio de Power (2013) se desarrolló en otro país, en concreto en Malasia.

Por otra parte, no se aprecian diferencias entre publicaciones australianas y estadounidenses con respecto a aspectos como la etapa educativa, la modalidad de ApS aplicado, las etapas educativas estudiadas o los métodos de investigación empleados. No obstante, sí existen diferencias relativas a los efectos que el ApS ocasiona sobre el alumnado implicado en su aplicación. En este sentido, es interesante mencionar que en los textos procedentes de Australia se aprecia un mayor valor al impacto del aprendizaje del alumnado relativo a las perspectivas cultural (Bartleet et al., 2016; Power, 2013) y política (Bartleet y Carfoot, 2013; Power, 2013), quizás por desarrollarse estos programas en ambientes culturales tan aislados y diferentes a lo que el estudiantado participante conoce.

En relación con lo que ya apuntaba la literatura científica previa, el meta-análisis de Yorio y Ye (2012) refrenda que el ApS se erige como un método pedagógico efectivo particularmente en programas académicos universitarios, puesto que han sido los más estudiados. En concreto, parece que la modalidad de servicio directo genera un mayor impacto en el estudiantado (Winterbottom, Lake, Ethridge, Kelly y Stubblefield, 2015), de manera que parece lógico que este tipo de servicio también sea el predominante en educación musical.

Por lo que respecta a la duración de las propuestas, imperan las que se prolongan durante un semestre académico, coincidiendo en muchos casos con la duración de la asignatura en la que se aplican (Bartolome, 2013). En todo caso, la gran mayoría de los textos describen programas con una duración de unas 40 horas o 30 semanas (un semestre), que son las duraciones que parecen aportar los mejores resultados (Conway et al., 2009).

Por otro lado, los resultados apuntan a que el ApS en educación musical prevalece en el área de la formación inicial docente; algo que ya fue señalado por Butin (2006). En este caso los contenidos principales contenidos a tratar por el alumnado se dividen en (1) relativos a la música o (2) concernientes a competencias genéricas relativas a la formación inicial docente. A tenor de lo expuesto en los textos analizados, los contenidos musicales a desarrollar son presentados al alumnado de manera clara y vinculados al servicio que se presta, tal y como aconsejan Yorio y Ye (2012).

Los entornos o colectivos receptores del servicio en proyectos de ApS coinciden en gran medida con otras propuestas socioeducativas que hacen uso de proyectos musicales comunitarios: personas mayores (ver, por ejemplo, Creech, Hallam, Varvarigou, McQueen y Gaunt, 2013; Formosa, 2013), espacios rurales (Nicholls y Sullivan, 
2016), prisiones y centros de detención (Cohen, 2009) o con comunidades indígenas (Marsh, 2000). Otros colectivos receptores de experiencias musicales comunitarias con fines sociales han incluido, entre otros, comunidades de personas migrantes y refugiadas (Broeske-Danielsen, 2013; Marsh, 2012), víctimas de conflictos armados o violencia directa y/o estructural (Rodríguez-Sánchez, Odena y Cabedo-Mas, 2018).

En general la idiosincrasia del ApS requiere la adopción de visiones de investigación de corte cualitativo, a fin de comprender el complejo fenómeno social que implica (Pérez-Juste, Galán y Quintanal, 2012). En el terreno musical la investigación sobre ApS concuerda con esta premisa, aunque se identifica un interés incipiente en el empleo de diseños mixtos, dado que su uso puede enriquecer la perspectiva de estudio (Salvador-García, Santágueda y Ruiz-Montero, 2018).

Acorde con los análisis cualitativos empleados mayoritariamente en educación musical, los instrumentos de investigación predominantes son las entrevistas, la observación y los diarios de reflexión, coincidiendo con las tres técnicas más recurrentes en los estudios previos de ApS (Rivera y Trigueros, 2018). Conviene mencionar en relación a este hecho que la reflexión emerge como un elemento básico para favorecer los aprendizajes del alumnado de ApS (Conway et al., 2009), por lo que el empleo de técnicas conversacionales y narrativas parece ser una buena opción no solo para la investigación per se, sino también para favorecer el aprendizaje.

En líneas generales, los programas de ApS han incorporado una serie de principios a las praxis educativas que han generado beneficios tanto a nivel social como en lo concerniente al desarrollo personal del alumnado. Sobre el impacto social generado, la literatura coincide al señalar que el ApS debe tener una orientación clara hacia la transformación de la sociedad (Rodríguez-Gallego, 2014), aunque los cambios generados no siempre son tan acentuados como se pretendía (Butin, 2006).

Con respecto al impacto generado sobre el alumnado que implementa los proyectos de ApS, la muestra estudiada incide en las cuatro perspectivas propuestas por Butin (2003). El enfoque técnico es el más recurrente en los estudios, incidiendo específicamente en los aprendizajes vinculados a la materia curricular (Conway et al., 2009), en este caso la música y/o la didáctica. En esta línea, una de las conclusiones que se extraen de los meta-análisis de Novak et al. (2007) y Warren (2012) es que el ApS resulta beneficioso para el aprendizaje de los objetivos curriculares. Como segunda perspectiva más repetida entre los artículos analizados emerge la cultural, que también ha sido subrayada como beneficio generado por el ApS (Yorio y Ye, 2012). Por último, las perspectivas política y post-estructural coinciden con tres únicas referencias, coincidiendo con las limitaciones que también muestra la literatura genérica sobre ApS. No obstante, tanto Conway et al. (2009) como Yorio y Ye (2012) ponen en valor diversas mejoras generadas en el estudiantado vinculadas con estos dos enfoques.

\section{Recomendaciones para la práctica y consideraciones finales}

Entendiendo el ApS en música como una propuesta que puede estar asentada en prácticas musicales socioeducativas, el estudio permite concluir que estas experiencias son adecuadas para el desarrollo de contenidos y aptitudes musicales en el estudiantado (Turino, 2008; Regelski, 2004). Como se desprende de la presente revisión el análisis nos lleva a negar la existencia de un único modelo de ApS capaz de generar beneficios 
a todos los estudiantes y comunidades de manera indiscriminada. Es decir, las diferencias entre los estudiantes, los colectivos receptores, la naturaleza de las necesidades abordadas y la idiosincrasia de las instituciones educativas implicadas, exigen necesariamente aproximaciones distintas a la hora de integrar el ApS en el currículum de educación musical. En consecuencia, los investigadores deberían ser muy cuidadosos en el diseño de investigaciones fundadas sobre diversos tipos de ApS, ajustándolas siempre a las características y necesidades de cada contexto (Chiva-Bartoll, 2018).

Los estudios analizados aluden a prácticas de ApS en la modalidad de servicio directo, evidenciando que esta modalidad facilita a los investigadores examinar el impacto de la participación en ApS sobre la adquisición de conocimientos académicos (Bartleet et al., 2016; Bartleet y Carfoot, 2013; Bartolome, 2013; Nicholls y Sullivan, 2016), valores conectados con los contenidos trabajados (Bartleet y Carfoot, 2013; Shepard y Booth, 2009) y cambios de actitud y/o comportamiento, tanto en el alumnado como en los colectivos receptores (Mcginnis y Mcdowell, 2003; Power, 2013). En este sentido, se identifica la necesidad de promover también propuestas de ApS a través de otras modalidades, con el fin de explorar los resultados de estas iniciativas en los aprendizajes musicales y en el desarrollo de competencias del estudiantado. Así, modelos de ApS que promuevan la interacción comunitaria pero que no impliquen una acción directa y sistemática sobre un colectivo externo al aula serían interesantes para ser desarrolladas especialmente en centros de educación primaria y secundaria.

También sería interesante profundizar sobre el compromiso y la motivación del alumnado hacia la educación musical cuando esta se vehicula a través de ApS; así como el diseño de más estudios longitudinales para despejar las dudas sobre los efectos que el ApS puede generar a largo plazo. Investigaciones previas en el campo de la educación musical y el desarrollo socioemocional indican que los potenciales beneficios de programas educativos dependen en parte de la longitud del desarrollo de estos programas, y sugieren que se requiere un promedio de al menos dos años antes de que se vea evidencia de beneficios (Campayo-Muñoz y Cabedo-Mas, 2017). Por ello consideramos que sería interesante estudiar el impacto de programas que articulen propuestas de ApS durante un periodo de tiempo sostenido, con el fin de identificar y contrastar mejor sus posibles beneficios.

Por otra parte, hablar de impacto social implica necesariamente evaluar los efectos que un programa educativo tiene en un contexto determinado. Las investigaciones estudiadas se han centrado principalmente en evaluar los efectos del ApS en el alumnado, por lo que incluir en el análisis una mayor presencia de la voz de la comunidad representa un reto para futuras investigaciones.

Para concluir, podemos decir que la educación musical empieza a asumir el desafío de afrontar, mediante métodos como el ApS, una praxis educativa sociocomunitaria en conexión con diversas necesidades sociales cercanas a los centros de enseñanza.

\section{Referencias bibliográficas}

Anderson, J., Swick, J. y Yff, J. (eds.). (2001). Service-learning in teacher education: enhancing the growth of new teachers, their students, and communities. Washington, DC: American Association of Colleges for Teacher Education.

Arrington, N. M. (2010). The effects of participating in a service-learning experience on the development of self-efficacy for self-regulated learning of third graders in an urban 
elementary school in southeastern United States. (Doctoral dissertation), Clemson University, Carolina del Sur, Estados Unidos de Norteamérica.

Bartleet, B. L., Sunderland, N. y Carfoot, G. (2016). Enhancing intercultural engagement through service learning and music making with Indigenous communities in Australia. Research Studies in Music Education, 38(2), 173-191. DOI: http://dx.doi. org/10.1177/1321103X16667863

Bartleet, B. L. y Carfoot, G. (2013). Desert harmony: Stories of collaboration between Indigenous musicians and university students. International Education Journal: Comparative Perspectives, 12(1), 180-196.

Bartolome, S. J. (2013). Growing through service: Exploring the impact of a service-learning experience on preservice educators. Journal of Music Teacher Education, 23(1), 79-91. DOI: http://dx.doi.org/10.1177/1057083712471951

Bartolome, S. J. (2017). Comparing Field-Teaching Experiences: A Longitudinal Examination of Preservice and First-Year Teacher Perspectives. Journal of Research in Music Education, 65(3), 264-286. DOI: http://dx.doi.org/10.1177/0022429417730043

Broeske-Danielsen, B. A. (2013). Community music activity in a refugee camp - student music teachers' practicum experiences. Music Education Research, 15(3), 304-316. https://doi.org/10.1080/14613808.2013.781145

Burton, S. y Reynolds, A. (2009). Transforming music teacher education through service learning. Journal of Music Teacher Education, 18(2), 18-33. DOI: http://dx.doi. org/10.1177/1057083708327872

Butin, D. W. (2003). Of what use is it? Multiple conceptualizations of service learning within education. Teachers College Record, 105(9), 1674-1692. DOI: http://dx.doi.org/10.1046/ j.1467-9620.2003.00305.x

Butin, D. W. (2006). The Limits of Service-Learning in Higher Education. The Review of Higher Education, 29(4), 473-498. DOI: http://dx.doi.org/10.1353/rhe.2006.0025

Cabedo-Mas, A., \& Díaz-Gómez, M. (2013). Positive musical experiences in education: Music as a social praxis. Music Education Research, 15(4), 455-470. doi: doi.org/10.108 0/14613808.2013.763780

Cabedo-Mas, A. (2014). La música como proyecto socioeducativo. En A. Giráldez (ed.), Didáctica de la música en primaria (pp. 101-120). Madrid, España: Editorial Síntesis.

Campayo-Muñoz, E. y Cabedo-Mas, A. (2017). The role of emotional skills in music education. British Journal of Music Education, 34(3), 243-258. DOI: http://dx.doi. org/10.1017/S0265051717000067

Casacuberta, D., Rubio, N. y Serra, L. (eds.). (2011). Acción cultural y desarrollo comunitario. Barcelona: Graó.

Chiva-Bartoll, O. (2018). Evaluación de proyectos de aprendizaje-servicio: porque lo que no se evalúa se devalúa. Revista Eufonía, Didáctica de la Música, 77, 30-36.

Chiva-Bartoll, O., Capella-Peris, C. y Pallarès-Piquer, M. (2018). Investigación-acción sobre un programa de aprendizaje-servicio en la didáctica de la educación física. Revista de Investigación Educativa, 36(1), 277-293.

Chiva-Bartoll, O., Pallarès-Piquer, M. y Gil-Gómez, J. (2018). Aprendizaje-servicio y mejora de la Personalidad Eficaz en futuros docentes de Educación Física. Revista Complutense de Educación, 29(1), 181-197.

Cohen, G. (2009). New theories and research findings on the positive influence of music and the art on health with ageing. Arts and Health,1(1), 48-62

Conway, J. M., Amel, E. L. y Gerwien, D. P. (2009). Teaching and Learning in the Social Context: A Meta-Analysis of Service Learning's Effects on Academic, Personal, Social, 
and Citizenship Outcomes. Teaching of Psicology, 36(4), 233-245. DOI: http://dx.doi. org/10.1080/00986280903172969

Cox, I. y Farmer, M. (2014). Competing with "Real Classes": The Value of Performing Ensemble Experiences for Non-Music Majors. En 121st ASEE AnnualConference and Exposition, Indianapolis, Estados Unidos de Norteamérica.

Creech, A., Hallam, S., Varvarigou, M., McQueen, H., y Gaunt, H. (2013). Active music making: a route to enhanced subjective well-being among older people. Perspectives in Public Health, 33(1). 36-43. https://doi.org/10.1177/1757913912466950

Doyle, M. A., Hotchkiss, G., Noel, M., Huss, A. y Holmes, R. (2004). Music informance as embodied service learning. The Journal of Educational Foundations, 18(2), 25-39.

Feen-Calligan, H. y Matthews, W. K. (2016). Pre-professional arts based service-learning in music education and art therapy. International Journal of Education \& the Arts, 17(17), $1-36$.

Formosa, M. (2013). Creativity in later life: Possibilities for personal empowerment. En A. Hansen, S. Kling and S. Strami González (Eds). Creativity, lifelong learning and the ageing population (pp.78-91). Östersund: Fornvårdaren.

Furco, A. (1996). Service-Learning: A balanced Approach to Experiential Education. Expanding Boundaries: Service and Learning. Washington DC: Corporation for National Service. 2-6.

Furco, A. (2002). Is service-learning really better than community service? A study of high school service program outcomes. En Furco, A. yBillig, S.H., Advances in servicelearning research: Vol.1. Service-learning: The essence of the pedagogy (pp. 23-50). Greenwich, CT.

Furco, A., \& Billig, S. H. (2002). Service Learning. The essence of the Pedagogy. Greenwich, Connecticut: Information Age Publishing.

Gil-Gómez, J., Moliner-García, O., Chiva-Bartoll, O. y García-López, R. (2016). Una experiencia de aprendizaje-servicio en futuros docentes. Revista Complutense de Educación, 27(1), 271-291.

Gregory, D. (2009). Online video bridges gap between orientation and first session for arts in medicine volunteers. Journal of music therapy, 46(4), 308-322. DOI: http://dx.doi. org/10.1093/jmt/46.4.308

Higgins, L. (2012). Community music: In theory and in practice.New York, Estados Unidos de Norteamérica: Oxford UniversityPress.

International Society for Music Education (2013): Community Music Activity Commission (CMA) Recuperado de: https://www.isme.org/our-work/commissions-forum/communitymusic-activity-commission-cma. [Consulta: agosto de 2018]

Marsh, K. (2000). Making connections: A case study of pre-service music education students' attitudinal change to Indigenous music. Research Studies in Music Education, 15, 58-67 https://doi.org/10.1177/1321103X0001500108

Marsh, K. (2012). "The beat will make you be courage": The role of a secondary school music program in supporting young refugees and newly arrived immigrants in Australia, Research Studies in Music Education, 34(2), 93-111. https://doi.org/10.1177/1321103X12466138

McGinnis, P. Y. y McDowell, D. (2003). Opera as an intervention for rural public school children. Academic Exchange Quarterly, 7(2), 178-184.

Menard, E. A. y Rosen, R. (2014). Preservice music teacher perceptions of mentoring young composers: an exploratory case study. Journal of Music Teacher Education, 25(2), 66-80. DOI: http://dx.doi.org/10.1177/1057083714552679

Miedl T. D. y Sulentic, M. M. (2018). Handbook of Research on Service-Learning Initiatives in Teacher Education Programs. Hershey, Estados Unidos de Norteamérica: IGI Global. 
Moher, D., Liberati, A., Tetzlaff, J. y Altman, D. G. (2009). Preferred reporting items for systematic reviews and meta-analyses: The PRISMA statement. BMJ,21, 331: b2535. DOI: http://dx.doi.org/10.1136/bmj.b2535

Nicholls, J. y Sullivan, B. M. (2016). Learning through dissonance: Critical service-learning in a juvenile detention center as field experience in music teacher education. Research Studies in Music Education, 38(2), 155-171. DOI: http://dx.doi.org/10.1177/1321103X16641845

Novak, J. M., Markey, V. y Allen, M. (2007). Evaluating cognitive outcomes of service learning in higher education: A meta-analysis. Communication Research Reports, 24(2), 149-157. DOI : http://dx.doi.org/10.1080/08824090701304881

Pérez-Juste, R., Galán, R. y Quintanal, J. (2012). Métodos y diseños de investigación en educación. Madrid, España: UNED- Universidad Nacional de Educación a Distancia.

Pike, P.D. (2017). Improving music teaching and learning through online service: A case study of a synchronous online teaching internship. International Journal of Music Education, 35(1), 107-117. DOI: http://dx.doi.org/10.1177/0255761415613534

Power, A. (2013). Developing the music pre-service teacher through international service learning. Australian Journal of Music Education, (2), 64-70.

Reynolds, A. M., Jerome, A., Preston, A. L. y Haynes, H. (2005). Service-learning in music education: Participants' reflections. Bulletin of the Council for Research in Music Education, 165, 79-91.

Rivera, E. y Trigueros, C. (2018). Metodología Cualitativa aplicada a la Investigación en Aprendizaje-Servicio. En O. Chiva y J. Gil (Eds.). Aprendizaje-servicio en la formación inicial docente: modelos de intervención e investigación (pp. 93-106). Barcelona, España: Octaedro.

Regelski, T.A. (2004). Social theory, and music and music education as praxis. Action, Criticism, and Theory for Music Education, 3(3), 2-52.

Rodríguez-Gallego, M.R. (2014). Service-learning as a methodological strategy at University. Revista Complutense de Educación, 25(1), 95-113.

Rodríguez-Sánchez, A., Odena, O., y Cabedo-Mas, A. (2018). Using life histories with sound postcards to investigate a music programme for social reconstruction in Colombia. British Journal of Music Education, 35(2), 177-190.

Salvador-García, C. y Ferrando-Félix, S. (2018). ¿Qué modalidades de ApS existen y cómo las uso en clase? Una aproximación práctica. Revista Eufonía. Didáctica de la música, 77, 37-40.

Salvador-García, C., Santágueda, M. y Ruiz-Montero, P. J. (2018). Posibilidades de los métodos cuantitativos y mixtos en la investigación del Aprendizaje-Servicio. En O. Chiva y J. Gil. (Eds.). Aprendizaje-servicio en la formación inicial docente (pp. 107118). Barcelona: Octaedro.

Seban, D. (2013). The impact of the type of projects on preservice teachers' conceptualization of service learning. Teaching and Teacher Education, 32, 87-97. DOI: http://dx.doi. org/10.1016/j.tate.2013.01.009

Shepard, J. y Booth, D. (2009). Heart to heart art. Reclaiming Children and Youth, 18(1), 12-15. Siebenaler, D. (2005). Music and service learning: A case study. Journal of Music Teacher Education, 15(1), 23-30. DOI: http://dx.doi.org/10.1177/10570837050150010105

Skinner, A. (2008). Global dimensions in service learning: A collaborative grant-writing project. International Education, 37(2), 6-16.

Turino, T. (2008). Music as Social Life: The Politics of Participation. Chicago, Estados Unidos de Norteamérica: Universityof Chicago Press. 
Vicente, G. y Azorín, C. M. (2013). Música y valores: una relación educativa ineludible. Música y Educación, 26(93), 16-25.

Warren, J. L. (2012). Does Service-Learning Increase Student Learning? A Meta-Analysis. Michigan Journal of Community Service Learning, 18(2), 56-61.

Winterbottom, C., Lake, V.E., Ethridge, E.A., Kelly, L., y Stubblefield, J.L. (2015). Fostering social justice through service-learning in early childhood teacher education. Revista Internacional de Educación para la Justicia Social, 2(2), 33-53.

Yorio, P. y Ye, F. (2012). A Meta-Analysis on the Effects of Service-Learning on the Social, Personal, and Cognitive Outcomes of Learning. Academy of Management Learning \& Education, 11(1), 9-27. DOI: http://dx.doi.org/10.5465/amle.2010.0072 